\title{
Primary Care Tracks in Medical Schools
}

Maribeth P. Williams, MD, MS | Denny Fe Agana, MPH | Benjamin J. Rooks, MS | Grant Harrell, MD

| Rosemary A. Klassen, BS | Robert Hatch, MD, MPH | Rebecca A. Malouin, PhD, MPH | Peter J.

Carek, MD, MS

PRIMER. 2019;3:3.

Published: 2/8/2019 | DOI: 10.22454/PRiMER.2019.799272

\section{Abstract}

Introduction: With the estimated future shortage of primary care physicians there is a need to recruit more medical students into family medicine. Longitudinal programs or primary care tracks in medical schools have been shown to successfully recruit students into primary care. The aim of this study was to examine the characteristics of primary care tracks in departments of family medicine.

Methods: Data were collected as part of the 2016 CERA Family Medicine Clerkship Director Survey. The survey included questions regarding the presence and description of available primary care tracks as well as the clerkship director's perception of impact. The survey was distributed via email to 125 US and 16 Canadian family medicine clerkship directors.

Results: The response rate was $86 \%$. Thirty-five respondents $(29 \%)$ reported offering a longitudinal primary care track. The majority of tracks select students on a competitive basis, are directed by family medicine educators, and include a wide variety of activities. Longitudinal experience in primary care ambulatory settings and primary care faculty mentorship were the most common activities. Almost $70 \%$ of clerkship directors believe there is a positive impact on students entering primary care.

Conclusions: The current tracks are diverse in what they offer and could be tailored to the missions of individual medical schools. The majority of clerkship directors reported that they do have a positive impact on students entering primary care.

\section{Introduction}

A 2014 US Institute of Medicine report highlighted the need for graduate medical education (GME) to produce more primary care physicians to care for underserved and rural populations. ${ }^{1}$ An estimated additional 52,000 primary care physicians will be needed within the next 10 years to meet the population's needs. ${ }^{2,3}$ The Council on GME estimates it will require $40 \%$ of practicing physicians to be in primary care to meet patient demands, which is an $8 \%$ increase from the current level. ${ }^{4,5}$

Suggestions for increasing student interest in family medicine include training students in high-performing ambulatory sites; creating a culture that values primary care, including population health education; and supporting primary care teaching faculty through awarding relative value units (RVUs) for precepting and teacher training, etc. ${ }^{4}$ Studies investigating student specialty choice have found several factors related to students choosing family medicine, including having a rural background, believing primary care is important, having low income earning expectations, attending a public medical school, and having positive faculty role models. ${ }^{6}$ Systematic reviews evaluating the impact of interventions aimed at recruiting students to a primary care career have found longitudinal 
programs with experiences in primary care and/or rural health were the only consistently successful interventions. ${ }^{3,7}$

Tracks are usually offered in addition to conventional curriculum and may include a variety of components. ${ }^{7}$ The aim of this study was to characterize longitudinal primary care tracks lasting at least 1 year, determine their prevalence, and estimate their impact on students entering primary care residencies. We hypothesized that while most medical schools do not offer longitudinal primary care tracks, clerkship directors (CD) at institutions where they are offered would feel they influence students to pursue primary care.

\section{Methods}

The questions of this study were administered to US and Canadian allopathic family medicine CDs as part of a larger omnibus survey conducted annually by the Council of Academic Family Medicine Educational Research Alliance (CERA). The survey methods and sample demographics have been presented previously. ${ }^{8}$

The CERA steering committee evaluated questions for consistency with the overall project aim, readability, and existing evidence of reliability and validity. Pretesting was done on family medicine educators who were not part of the target population. Questions were modified following pretesting for flow, timing, and readability. The American Academy of Family Physicians Institutional Review Board (IRB) and the University of Florida IRB approved the study. Data were collected between August 9, 2016 and October 9, 2016.

The sampling frame for the survey was institutional representatives-such as the CD or their designee-of allopathic medical schools. Email invitations to participate were delivered with the survey utilizing the online program SurveyMonkey. Four follow-up emails were sent after the initial email invitation to encourage nonrespondents to participate.

\section{Survey Items}

The survey collected basic demographic information about CDs and their medical schools. Survey questions asked about the presence and description of available primary care tracks as well as the CD's perception of impact (Table 1). Additional questions were based on literature review as well as the research team's knowledge of activities offered in medical schools (Table 2). A primary care track was defined in the survey as "formal, structured activities that occurred for a period longer than 1 year and were led by an identified faculty member." We did not further define what activities were considered as primary care.

\section{Statistical Analysis}

Descriptive statistics were used to characterize demographic data and longitudinal primary care tracks. Logistic regression was used to investigate associations between schools with primary care tracks and those without across the variety of variables present. The same approach was used to consider associations between programs planning to implement a primary care track in our data. In order to account for the effects of multiple comparison testing on our results, $P$ values were corrected using the false discovery rate approach where appropriate. ${ }^{9}$ Fisher exact tests were used to test for associations among the subgroup of institutions with a primary care track. Associations between aspects of the track and clerkship directors' perceptions on the impact of their primary care track on applications to primary care residencies were investigated. Multiple comparison corrections were not applied to this set of tests due to the greater probability of a false negative as a result of a smaller sample size. All analyses were done using $\mathrm{R}$ version 3.2.2 statistical program.

\section{Results}

The survey was distributed via email to 125 US and 16 Canadian family medicine CDs, with a response rate of $86 \%$. Thirty-five respondents (29\%) reported currently offering a longitudinal primary care track (Table 1 ). The greatest barriers to offering a longitudinal track included lack of medical school support (34\%) and funded faculty time (29\%). Fourteen schools (16\%) planned to offer one in the next 1-2 years (Table 1). There was great variation in activities, but nearly all included a longitudinal primary care ambulatory clinical experience and provided a primary 
care faculty mentor (Table 2). Community engagement as well as additional didactic sessions were also present in most tracks (Table 2). Almost $70 \%$ of CDs felt there was a positive impact on students entering primary care (Table 1). There were no significant differences in characteristics (eg, school region, public or private school, CD gender or ethnicity) between schools with primary care tracks and those without, or between programs planning to implement a primary care track and those that weren't. Also, CDs' perceptions of the impact of their program on the number of students applying to primary care programs was not associated with the program offerings (Table 2) or what year students begin their clerkship rotations.

\section{Discussion}

Longitudinal primary care tracks are varied in their characteristics. They are currently offered in about one-third of medical schools with another $10 \%$ planned in upcoming years. However, a lack of faculty and/or medical school support were barriers, which may suggest that colleges of medicine are not as supportive of these tracks.

Our study had several limitations. The primary care track impact on students applying to primary care residencies was measured based on the CDs' perceptions, and such perceptions may be inaccurate. We are unable to determine the actual impact of the track on residency selection. In addition, the survey was only given to CDs in family medicine who may not have been aware of primary care tracks based in other departments. The survey definition of primary care tracks may have been unclear, as we did not clearly state the activities in the structured curriculum should be related to primary care, and some respondents may not have considered other tracks at their institutions as primary care.

Overall this study found that primary care longitudinal tracks are diverse in structure and characteristics, which could allow them to be tailored to meet the missions of individual medical schools. If such tracks have a significant positive impact on students entering primary care and the need for primary care physicians remains at such high levels, a national primary care education organization (eg, Society of Teachers of Family Medicine) should provide a standardized set of criteria or suggestions for such tracks in all medical schools. Additional studies are needed to verify and support this claim.

\section{Tables and Figures}


Table 1: Descriptive Statistics of Primary Care Track Survey Questions

\begin{tabular}{|c|c|}
\hline Survey Question & $\begin{array}{l}\text { Number of } \\
\text { Respondents (\%) }\end{array}$ \\
\hline Currently offer a primary care track? & $\mathrm{N}=120$ \\
\hline Yes & $35(29.2)$ \\
\hline If "No" to \#1, why not? & $\mathrm{N}=84$ \\
\hline Medical school is not supportive & $29(34.5)$ \\
\hline Not enough funded faculty time to create/lead one & $25(29.8)$ \\
\hline Never considered it & $16(19.0)$ \\
\hline Plan to start one in the next $1-2$ years & $14(16.7)$ \\
\hline How are students selected? & $N=30$ \\
\hline Competitive application process as part of application to medical school & $14(46.7)$ \\
\hline Competitive application process once accepted to medical school & $9(30.0)$ \\
\hline Open to all interested students & $7(23.3)$ \\
\hline What year do students typically begin participation? & $\mathrm{N}=35$ \\
\hline M1 & $29(82.9)$ \\
\hline M2 & $7(20.0)$ \\
\hline M3 & $8(22.9)$ \\
\hline M4 & $4(11.4)$ \\
\hline How long (in academic years) has your primary care track been in existence? & 8.1 (mean) \\
\hline Please select which group primarily is in charge of the primary care track: & $N=33$ \\
\hline Family medicine department & $25(75.8)$ \\
\hline Internal medicine department & $8(24.2)$ \\
\hline Do faculty have protected / paid time specifically for tasks related to the primary care track? & $N=35$ \\
\hline Yes & $25(71.4)$ \\
\hline $\begin{array}{l}\text { What impact do you believe the primary care track has had on the number of students applying to primary } \\
\text { care residencies (IM, FM, Peds): }\end{array}$ & $N=33$ \\
\hline Strong negative impact & $1(3.0)$ \\
\hline Slight negative impact & $0(0.0)$ \\
\hline Neutral impact & $9(27.3)$ \\
\hline Slight positive impact & $7(21.2)$ \\
\hline Strong positive impact & $16(48.5)$ \\
\hline
\end{tabular}


Table 2: Descriptive Statistics of Primary Care Track Activities

\begin{tabular}{|c|c|}
\hline Survey Question & $\begin{array}{c}\text { Number of } \\
\text { Respondents (\%) }\end{array}$ \\
\hline Which of the following activities have been integrated in the primary care track? & $\mathrm{N}=35$ programs \\
\hline Longitudinal clinical experience in a primary care ambulatory setting & $33(94.3)$ \\
\hline Primary care faculty mentor & $30(85.7)$ \\
\hline Community engagement & $21(60.0)$ \\
\hline Additional didactic sessions including invited speakers or clinical instruction & $18(51.4)$ \\
\hline Journal and/or book clubs & $14(40.0)$ \\
\hline Population health management & $14(40.0)$ \\
\hline Allocated time for track curriculum built into student schedules & $12(34.3)$ \\
\hline Procedure workshops or seminars & $11(31.4)$ \\
\hline Funding for primary care meeting/conference or similar activity & $9(25.7)$ \\
\hline Research component & $8(22.9)$ \\
\hline Quality improvement & $7(20.0)$ \\
\hline Leadership training & $6(17.1)$ \\
\hline Scholarship/stipend for participation & $6(17.1)$ \\
\hline Student-run primary care clinic & $6(17.1)$ \\
\hline Guaranteed residency spot through completion of the track & $4(11.4)$ \\
\hline Health policy training & $4(11.4)$ \\
\hline Average number of components per track & 5.8 \\
\hline
\end{tabular}

\section{Acknowledgments}

Presentations: Agana DF, Carek P, Harrell J, et al. Primary Care Tracks in Medical Schools: A CERA Survey. Presentation at Society of Teachers of Family Medicine in Washington, DC; May 2018.

\section{Corresponding Author}

Maribeth P. Williams, MD, MS

University of Florida, Department of Community Health and Family Medicine, PO Box 100237, Gainesville, FL 32610. 352-273-5976. Fax: 352-273-5213.

maribethporter@ufl.edu

\section{Author Affiliations}

Maribeth P. Williams, MD, MS - University of Florida Department of Community Health and Family Medicine Denny Fe Agana, MPH - Department of Community Health and Family Medicine, University of Florida, Gainesville Benjamin J. Rooks, MS - Department of Community Health and Family Medicine, University of Florida Grant Harrell, MD - Department of Community Health and Family Medicine, University of Florida Rosemary A. Klassen, BS - University of Florida College of Medicine Robert Hatch, MD, MPH - Department of Community Health and Family Medicine, University of Florida Rebecca A. Malouin, PhD, MPH - Department of Pediatrics and Human Development, Michigan State University Peter J. Carek, MD, MS - Department of Community Health and Family Medicine, College of Medicine, University of Florida

\section{References}

1. Institute of Medicine. Graduate Medical Education That Meets the Nation's Health Needs. Washington, DC: 
The National Academies Press; 2014. https://doi.org/10.17226/18754

2. Petterson SM, Liaw WR, Phillips RL Jr, Rabin DL, Meyers DS, Bazemore AW. Projecting US primary care physician workforce needs: 2010-2025. Ann Fam Med. 2012;10(6):503-509. https://doi.org/10.1370 /afm.1431

3. Dupras DM, West CP. Training for Careers in Primary Care: Time for Attention to Culture. J Gen Intern Med. 2015;30(9):1243-1244. https://doi.org/10.1007/s11606-015-3416-1

4. Kernan WN, Elnicki DM, Hauer KE. The Selling of Primary Care 2015. J Gen Intern Med. 2015;30(9):1376-1380. https://doi.org/10.1007/s11606-015-3364-9

5. Kozakowski SM, Crosley PW, Bentley A. Results of the 2014 National Resident Matching Program ${ }^{\circledR}$ : family medicine. Fam Med. 2014;46(9):701-706.

6. Senf JH, Campos-Outcalt D, Kutob R. Factors related to the choice of family medicine: a reassessment and literature review. J Am Board Fam Pract. 2003;16(6):502-512. https://doi.org/10.3122/jabfm.16.6.502

7. Pfarrwaller $E$, Sommer J, Chung C, et al. Impact of Interventions to Increase the Proportion of Medical Students Choosing a Primary Care Career: A Systematic Review. J Gen Intern Med. 2015;30(9):1349-1358. https://doi.org/10.1007/s11606-015-3372-9

8. Mainous AG III, Seehusen D, Shokar N. CAFM Educational Research Alliance (CERA) 2011 Residency Director survey: background, methods, and respondent characteristics. Fam Med. 2012;44(10):691-693.

9. Benjamini $Y$, Hochberg $Y$. Controlling the false discovery rate: a practical and powerful approach to multiple testing. J R Stat Soc B. 1995;57(1):289-300. https://doi.org/10.1111/j.2517-6161.1995.tb02031.x

Copyright $\odot 2019$ by the Society of Teachers of Family Medicine 